\title{
Bénard Problem for Slightly Compressible Fluids: Existence and Nonlinear Stability in 3D
}

\author{
Arianna Passerini \\ Department of Mathematics and Computer Science, University of Ferrara, Via Machiavelli 30, 40121 Ferrara, Italy \\ Correspondence should be addressed to Arianna Passerini; ari@unife.it
}

Received 5 May 2020; Accepted 19 June 2020; Published 8 July 2020

Academic Editor: Mayer Humi

Copyright (C) 2020 Arianna Passerini. This is an open access article distributed under the Creative Commons Attribution License, which permits unrestricted use, distribution, and reproduction in any medium, provided the original work is properly cited.

This paper shows the existence, uniqueness, and asymptotic behavior in time of regular solutions (a la Ladyzhenskaya) to the Bénard problem for a heat-conducting fluid model generalizing the classical Oberbeck-Boussinesq one. The novelty of this model, introduced by Corli and Passerini, 2019, and Passerini and Ruggeri, 2014, consists in allowing the density of the fluid to also depend on the pressure field, which, as shown by Passerini and Ruggeri, 2014, is a necessary request from a thermodynamic viewpoint when dealing with convective problems. This property adds to the problem a rather interesting mathematical challenge that is not encountered in the classical model, thus requiring a new approach for its resolution.

\section{Introduction}

As is well known, one of the most accepted models in the study of convection problems in fluid mechanics is the socalled Oberbeck-Boussinesq approximation (hereafter denoted by $\mathrm{O}-\mathrm{B}$ ) [1-5]. The latter is characterized by the fact that one keeps the fluid incompressible and allows for density changes only in the buoyancy term of the linear momentum equation, by assuming a linear dependence on temperature.

However, as observed in [6] and further elaborated in [7], O-B may not be entirely satisfactory or even consistent from the physical viewpoint [8] if the density is not allowed to have a dependence also on the pressure field $p$. As a result, in [9], a more general theory aimed at removing this incongruence was proposed. More specifically, while still keeping the incompressibility constraint, the density in the buoyancy term was allowed to depend, linearly, also on $p$. The remarkable feature of this new model is that now, unlike the classical $\mathrm{O}-\mathrm{B}$, the linear momentum equation contains a pressure term that is not in the classical gradient form. From a mathematical perspective, this feature is very challenging in that some fundamental estimates necessary to ensure stability properties and well-posedness of the relevant problems $[10,11]$ no longer hold and, consequently, the study of such problems requires fresh ideas and different approach.

With this in mind, a systematic study of the mathematical properties of the new model was started, focusing on the classical Bénard problem. As is well known, the latter regards convective motions stemming from a motionless basic flow, $\mathbf{r}_{0}$, of a horizontal layer of fluid heated from below. For the new model, the state $\mathbf{r}_{0}$ is given by [7]

$$
\begin{aligned}
& \mathbf{v} \equiv 0, \\
& T(z) \equiv T_{r}(z)=T_{0}-\frac{\delta T}{h} z, \\
& p(z) \equiv p_{r}(z)=p_{0}+p_{b} e^{-\rho_{0} g \beta z}+\frac{1}{\beta^{2}} \frac{\alpha \delta T}{\rho_{0} g h}\left(1-e^{-\rho_{0} g \beta z}\right) \\
&-\frac{1}{\beta}\left(1-e^{-\rho_{0} g \beta z}+\frac{\alpha \delta T}{h} z\right),
\end{aligned}
$$

with the $z$ coordinate in the vertical direction. Here, $\mathbf{v}, T$, and $p$ are the velocity, temperature, and pressure fields, respectively, $\rho_{0}$ and $p_{0}$ are the (constant) gauge density and pressure, $T_{0}$ and $T_{0}+\delta T$ are the temperature at bottom and 
top of the layer, and $h$ is the thickness of the layer. Finally, $\alpha$ and $\beta$ are the positive material constants. Notice that (1) tends to the motionless solution for the same problem in the $\mathrm{O}-\mathrm{B}$ approximation by letting $\beta \longrightarrow 0$. In $[4,9,12]$, the linear and nonlinear stability of $\mathbf{r}_{0}$, as well as the existence and uniqueness for the associated "perturbed problem," was investigated. All this work was done in the case of the plane flow.

The objective of the current paper is to investigate the same problem in the more general and appropriate threedimensional setting. The approach followed here to overcome the problem of the pressure mentioned earlier on is to introduce a new equation of the elliptic type for the pressure field, disregarding the incompressibility constraint (for steady and for nonlinear equations close to the one of nonNewtonian fluids, this approach that allows to achieve a posteriori the divergence-free equation was considered in [5]. Actually, although this work is done in a "parabolic context" (for the kinetic field), the technique in [5] in some sense has inspired the approach in the present paper). In such a way, the original system of equations is converted to three highly nonlinearly coupled equations for velocity, temperature, and pressure fields. However, for this new problem, it is shown that if the velocity field, $\mathbf{v}$, is sufficiently smooth and also solenoidal at time $t=0$, then $\mathbf{v}$ must be solenoidal in the entire interval of time where the solution exists, thus providing a solution to the original problem. In this way, it is shown that, if $\beta<2 \pi$, the relevant perturbed problem (to the state $\mathbf{r}_{0}$ ) has a local, strong solution (a la Ladyzhenskaya) corresponding to initial data of arbitrary size (in a suitable Sobolev class). Such a solution becomes, in fact, global if this size is appropriately restricted and the Rayleigh number is below a certain value, see Theorem 1 .

The plan of the paper is given in the following. After formulating the problem and collecting some preliminary results in Section 1, in Section 2, the classical Galerkin method is used (see, for instance, [4]) to construct an "approximate" solution. In Section 3, which constitutes the main core of this paper, a number of fundamental estimates on the approximate solution is proven that, again by classical techniques, allows us to conclude that the latter converges to a solution of the original problem having the properties described above.

\section{Notations and Preliminary Results}

The flow domain is an infinite, horizontal layer bounded in the vertical $z$-direction with thickness $h$ and unbounded and invariant in the $x$ and $y$ directions. It is assumed that the generic perturbed field is periodic in $x$ and $y$ of a given period $L_{x}$ and $L_{y}$, respectively, an assumption that is justified by the fact that the convective roll pattern has a periodic structure. The relevant spatial region thus becomes a "periodicity cell" defined (in nondimensional form) as

$$
\Omega_{0}=(0, a) \times(0, b) \times(0,1),
$$

with $a=L_{x} / h$ and $b=L_{y} / h$. Just for simplicity, set, herein, $a=b=1$. The mean value of a function $f$ is indicated as

$$
\langle f\rangle=\frac{1}{\left|\Omega_{0}\right|} \int_{\Omega_{0}} f(x, y, z, t) \mathrm{d} x \mathrm{~d} y \mathrm{~d} z,
$$

and a partial derivative with a subscript $\partial_{\xi} \phi=(\partial \phi / \partial \xi)=\phi_{\xi}$ with $\xi \in\{x, y, z, t\}$, and similarly for higher-order derivatives. As customary, the inner product in $L^{2}\left(\Omega_{0}\right)$ is denoted by

$$
(\mathbf{u}, \mathbf{v}):=\int_{\Omega_{0}} \mathbf{u} \cdot \mathbf{v} \mathrm{d} x \mathrm{~d} y \mathrm{~d} z,
$$

where $\mathbf{u}$ and $\mathbf{v}$ denote the scalar vector or tensor fields. The components of a vector $\mathbf{u}$ are denoted by $\mathbf{u} \equiv\left(u^{x}, u^{y}, u^{z}\right)$. Let $W^{m, p}\left(\Omega_{0}\right)$ be the usual Sobolev space and $\|u(t)\|_{m, p}$ be the associated norm. The subspace of $W^{m, p}\left(\Omega_{0}\right)$ constituted by those $u$ with $\langle u\rangle=0$ is denoted by $\mathscr{W}^{m, p}\left(\Omega_{0}\right)$. By $L^{\infty}\left((0, T) ; W^{m, p}\left(\Omega_{0}\right)\right)$, we denote the space of functions $u$ such that

$$
|u|_{\infty, m, p}:=e s s \sup _{t \in[0, T]}\|u(t)\|_{m, p}<\infty .
$$

Likewise, for $q \in[1, \infty)$, the Bochner space $L^{q}\left((0, T) ; W^{m, p}\left(\Omega_{0}\right)\right)$ is the space defined by the norm

$$
|u|_{q, m, p}:=\left(\int_{0}^{T}\|u(t)\|_{m, p}^{q} \mathrm{~d} x\right)^{1 / q}<\infty .
$$

A decisive role in our stability analysis is played by the Poincaré inequality in $\Omega_{0}$ :

$$
\|u\|_{2} \leq C\|\nabla u\|_{2} .
$$

As is known (see [13]), set $\Gamma=\bar{\Omega}_{0} \cap\{z=0,1\}$; this inequality is true if at least one of the following cases is satisfied: (a) functions with a zero mean value, (b) vector functions with a vanishing normal component on $\Gamma$, and (c) scalar functions vanishing on $\Gamma$. Throughout, the same symbol $C$ is used to denote the involved constant, which depends only on the domain. Since boundary conditions are used for which the following integration by parts is valid:

$$
\|\nabla u\|_{2}=-(\Delta u, u),
$$

if the Poincaré inequality holds true for $u$, then by the Schwarz inequality, it also follows

$$
\|\nabla u\|_{2} \leq C\|\Delta u\|_{2} .
$$

As a matter of fact, in $\Omega_{0}$, there is an equivalence between the norm of the Laplacian and that of the full set of the second derivatives, as shown by Ladyzhenskaya (see [14], equations (17) and (18) at page 18]):

$$
\|\Delta u\|_{2}=\left\|D^{2} u\right\|_{2} .
$$

Further tools, herein suitable, are some typical 3D immersion inequalities: the Sobolev-Gagliardo-Nirenberg inequality:

$$
\|u\|_{6} \leq C\|u\|_{1,2},
$$

which in case the Poincaré inequality also holds true becomes 


$$
\begin{gathered}
\|u\|_{6} \leq C\|\nabla u\|_{2}, \\
\|\nabla u\|_{6} \leq C\|\Delta u\|_{2} .
\end{gathered}
$$

In what follows, Morrey's inequality is also used (still simplified by the Poincaré inequality):

$$
\|u\|_{\infty} \leq C\|\nabla u\|_{6} .
$$

A further preliminary result consists in the estimates for the Laplace problem (e.g., $[15,16])$.

Lemma 1. Suppose $u$ is a weak solution to

$\Delta u=f$ in $\Omega_{0}$ with either (a) $u=0$ or (b) $\partial_{z} u=0$ at $z=0,1$.

Then, if $f \in W^{m, 2}\left(\Omega_{0}\right), m \geq 0$, one has $u \in W^{m+2,2}\left(\Omega_{0}\right)$ in case (a), and the following inequality holds:

$$
\|u\|_{m+2,2} \leq c\|f\|_{m, 2}
$$

with $c$ independent of $u$ and $f$. The same result holds in case (b) with $u \in \mathscr{W}^{m+2,2}\left(\Omega_{0}\right)$, provided $f \in \mathscr{W}^{m, 2}\left(\Omega_{0}\right)$.

In the present framework, once the perturbation fields $\tau=T-T_{r}$ and $P=p-p_{r}$ are defined, the following nondimensional system of equations are addressed:

$$
\left\{\begin{array}{l}
\nabla \cdot \mathbf{v}=0 \\
\frac{1}{\operatorname{Pr}}\left(\frac{\partial \mathbf{v}}{\partial t}+\mathbf{v} \cdot \nabla \mathbf{v}\right)=-\nabla P-\beta P \mathbf{k}+\Delta \mathbf{v}+\operatorname{Ra} \tau \mathbf{k} \\
\frac{\partial \tau}{\partial t}+\mathbf{v} \cdot \nabla \tau=\Delta \tau+\mathbf{v} \cdot \mathbf{k}
\end{array}\right.
$$

where $\mathrm{Ra}$ and $\mathrm{Pr}$ are the Rayleigh and Prandtl numbers, respectively, and $\mathbf{k}=(0,0,1)$ is directed along the upward vertical direction. Notice that, given $p_{0}$, the perturbation $P$ has to be zero at the reference state. The boundary conditions are homogeneous for $\tau$, while for $\mathbf{v}$, one imposes the impermeability condition $v^{z}=0$ and the stress-free condition $v_{z}^{x}=v_{z}^{y}=0$, so that one could compare the stability results with the classic exact ones obtained under the same conditions. Let us set, just for simplicity, $\nabla \cdot \mathbf{v}=\phi$.

For "regular" solutions, i.e., solutions, such that

$$
\begin{aligned}
& \mathbf{v} \in L^{\infty}\left((0, T) ; W^{2,2}\left(\Omega_{0}\right)\right) \cap L^{2}\left(0, T ; W^{3,2}\left(\Omega_{0}\right)\right), \\
& \mathbf{v}_{t} \in L^{\infty}\left((0, T) ; L^{2}\left(\Omega_{0}\right)\right) \cap L^{2}\left(0, T ; W^{1,2}\left(\Omega_{0}\right)\right),
\end{aligned}
$$

corresponding to $\mathbf{v}_{0} \in W^{2,2}\left(\Omega_{0}\right)$ at the initial time, one can show that system (17) is equivalent to

$$
\left\{\begin{array}{l}
\Delta P+\beta P_{z}=-\frac{1}{\operatorname{Pr}} \nabla \cdot(\mathbf{v} \cdot \nabla \mathbf{v}+\phi \mathbf{v})+\operatorname{Ra} \tau_{z} \\
\frac{1}{\operatorname{Pr}}\left(\frac{\partial \mathbf{v}}{\partial t}+\mathbf{v} \cdot \nabla \mathbf{v}+\phi \mathbf{v}\right)=-\nabla P-\beta P \mathbf{k}+\Delta v+\operatorname{Ra} \tau \mathbf{k} \\
\frac{\partial \tau}{\partial t}+\mathbf{v} \cdot \nabla \tau=\Delta \tau+\mathbf{v} \cdot \mathbf{k}
\end{array}\right.
$$

with Robin's conditions for $P$ at $z=0,1$ :

$$
\nabla P \cdot \mathbf{k}+\beta P=0 .
$$

The extra nonlinear term lying at the left-hand side of $(19)_{2}$ is inserted to make the stress-free condition compatible with the equation, by matching the mean value of the horizontal components. As a matter of fact, $\mathbf{v} \cdot \nabla \mathbf{v}+\phi \mathbf{v}=$ $\nabla \cdot(\mathbf{v} \otimes \mathbf{v})$ gives rise, once integrated, to a vanishing boundary integral, as any other terms in the horizontal projection of equation $(19)_{2}$.

Actually, in one direction, the implication is obvious: it is sufficient to take the divergence of the second equation in (17) to obtain the first of (19). The boundary conditions for $P$ are established by evaluating at $z=0,1$ the normal component of $(17)_{2}$ : the trace of $v_{t}^{z}$ and, by regularity, the trace of $v_{z z}^{z}$ are defined and vanished, leading to (20). As a matter of fact, by deriving the divergence-free condition with respect to $z$, one gets $v_{z x}^{x}+v_{z y}^{y}+v_{z z}^{z}=0$, and deriving the stress-free condition along the boundary (since one can exchange the order of derivation), one gets $v_{z x}^{x}=v_{x z}^{x}=0$ and $v_{z y}^{y}=v_{y z}^{y}=0$, which can be replaced in the previous one.

Conversely, let us assume that (19) holds true and the solutions are regular; in particular, assume that $P \in L^{2}\left(0, T ; W^{2,2}\left(\Omega_{0}\right)\right)$. Let us replace $(19)_{1}$ in the divergence of $(19)_{2}$, then one obtains

$$
\frac{1}{\operatorname{Pr}} \frac{\partial \phi}{\partial t}=\Delta \phi
$$

Let us remark that $\langle\phi\rangle=0$. Indeed, if $v_{x}^{x}$ or $v_{y}^{y}$ is different from zero, then their mean value is zero by periodicity. So, one deduces $\langle\phi\rangle=\left\langle v_{z}^{z}\right\rangle=0$ because after integration in $z \in(0,1)$, one obtains $v^{z}$. As a consequence, one can use the Poincaré inequality for $\phi$.

Next, one can perform the $L^{2}$-inner product of the equation with $\phi$ : by taking into account the periodicity conditions, the right-hand side of (21) becomes

$$
\begin{aligned}
(\Delta \phi, \phi)= & \frac{1}{2} \int_{0}^{1} \mathrm{~d} x \int_{0}^{1} \mathrm{~d} y\left[\left(\phi^{2}\right)_{z}(x, y, 1)-\left(\phi^{2}\right)_{z}(x, y, 0)\right] \\
& -(\nabla \phi, \nabla \phi),
\end{aligned}
$$

from the regularity hypotheses, one can deduce that the boundary terms vanish in the trace sense because of impermeability and stress-free conditions, which implies $v_{z x}^{x}=$ $v_{x z}^{x}=0$ and $v_{z y}^{y}=v_{y z}^{y}=0$. On the other hand, Robin's condition for the pressure implies $\Delta v^{z}=0$ in the momentum balance at the boundary. Hence, since one can derive the impermeability condition with respect to $x$ and $y$, it follows also $v_{z z}^{z}=0$. Thus, by summing up, one sees that $\phi_{z}$ vanishes at the boundaries.

In conclusion, one can write

$$
\frac{1}{\operatorname{Pr}} \frac{\mathrm{d}}{\mathrm{d} t}\|\phi\|_{2}^{2}+\|\nabla \phi\|_{2}^{2} \leq 0 .
$$

Finally, either the divergence of the velocity is zero at $t=0$ and then for all $t>0$, or it decays exponentially to zero. 
This means that the divergence-free condition can be fulfilled and is, moreover, physically observable.

Hereafter, a solution with the required regularity is shown to exist, provided the initial data for $(19)_{2}$ and $(19)_{3}$ are in $W^{2,2}\left(\Omega_{0}\right)$.

Concerning boundary conditions, the change of variable $\Pi=P e^{\beta z}$ turns Robin's ones into the simpler Neumann conditions:

$$
\Pi_{z}=0, \quad z=0,1,
$$

and simplifies the system under study, which becomes like the one proposed in [9], page. 7, and studied in [4]:

$$
\left\{\begin{array}{l}
\Delta \Pi-\beta \Pi_{z}=-\frac{e^{\beta z}}{\operatorname{Pr}} \nabla \cdot(\mathbf{v} \cdot \nabla \mathbf{v}+\phi \mathbf{v})+\operatorname{Ra} e^{\beta z} \tau_{z} \\
\frac{1}{\operatorname{Pr}}\left(\frac{\partial \mathbf{v}}{\partial t}+\mathbf{v} \cdot \nabla \mathbf{v}+\phi \mathbf{v}\right)-\Delta \mathbf{v}=-\nabla\left(e^{-\beta z} \Pi\right)-\beta e^{-\beta z} \Pi \mathbf{k} \\
\quad+\operatorname{Ra} \tau \mathbf{k}, \\
\frac{\partial \tau}{\partial t}+\mathbf{v} \cdot \nabla \tau-\Delta \tau=\mathbf{v} \cdot \mathbf{k} .
\end{array}\right.
$$

The new variable $\Pi$ is defined up to an arbitrary constant, unlike $P$ which appears in the buoyancy force and depends on the boundary value $p_{b}$ which is in the basic solution. Thus, $\Pi$ can be prescribed with a mean value zero and verify the Poincaré inequality.

The trivial solution $\left(\mathbf{v}=c_{1} \mathbf{i}+c_{2} \mathbf{j}, \tau=0, P=0\right)$ corresponding to the Galilean invariance has to be considered equivalent to the rest state, so for this reason, solutions are looked in Banach subspaces defined by the following condition:

$$
\left\langle v^{x}\right\rangle=\left\langle v^{y}\right\rangle=0 .
$$

Next, one assigns in $\Omega_{0}$ the periodicity conditions in the horizontal directions and the stress-free conditions at the boundaries and homogeneous conditions for the deviatory temperature $\tau$ :

$$
\begin{aligned}
\Pi_{z}(x, y, 0, t) & =\Pi_{z}(x, y, 1, t)=0, \\
v^{z}(x, y, 0, t) & =v^{z}(x, y, 1, t)=0, \\
v_{z}^{x}(x, y, 0, t) & =v_{z}^{x}(x, y, 1, t)=0, \\
\tau(x, y, 0, t) & =\tau(x, y, 1, t)=0 .
\end{aligned}
$$

At the initial time, data are given concordant with the previous ones:

$$
(\mathbf{v}(x, y, z, 0), \tau(x, y, z, 0))=\left(\mathbf{v}_{0}(x, y, z), \tau_{0}(x, y, z)\right) .
$$

The plan is studying (17) before proving the equivalence with (25). To this end, one looks for solutions such that a.e. in $t$, and for $k \in \mathbb{N}_{0}$, $\Pi$ belongs to $W^{k, 2}\left(\Omega_{0}\right)$, which is defined as the closure in the Sobolev space norm of $C^{\infty}\left(\Omega_{0}\right)$, whose functions are periodic in $x$ and $y$, verifies the homogeneous Neumann condition at $z=0,1$, and has a zero mean value (this last feature is necessary to prove the existence).
Analogously, without the isochoric conditions, $\mathbf{v} \equiv\left(v^{x}, v^{y}, v^{z}\right)$ has free components such that $v^{x}$ and $v^{y}$ belong to $\mathscr{W}^{k, 2}\left(\Omega_{0}\right)$ (with the mean value zero, as previously noticed), while $v^{z}$ and $\tau$ belong to $W_{0}^{k, 2}\left(\Omega_{0}\right)$, which is the closure of $C_{0}^{\infty}\left(\Omega_{0}\right)$.

\section{Galerkin Approximation}

Still in [9] and in [4], an existence result for $\Pi$ and further estimates are given in $2 \mathrm{D}$. The proof is unaffected by the dimension, and the results are summarized below.

Lemma 2. If $0<\beta<2 \pi$ and if $f \in L^{2}\left(\Omega_{0}\right)$, then the problem

$$
\begin{cases}\Delta \Pi-\beta \Pi_{z}=e^{\beta z} f, & \text { in } \Omega_{0}, \\ \Pi_{z}(x, y, 0)=\Pi_{z}(x, y, 1)=0, & \text { for }(x, y) \in \mathbb{R}^{2},\end{cases}
$$

with periodic side conditions, has a unique solution $\Pi \in \mathscr{W}^{2,2}\left(\Omega_{0}\right)$ such that $\langle\Pi\rangle=0$, and the following estimate holds true:

$$
\|\Pi\|_{2,2} \leq C(\beta)\|f\|_{2}
$$

In this lemma and hereafter, $C(\beta)$ stands for a positive function of $\beta$ such that $\lim _{\beta \longrightarrow 0} C(\beta)=c \geq 0$. The same convention is kept for $C(\beta, \operatorname{Pr}, \mathrm{Ra})$.

Furthermore, if $f=\nabla \cdot \mathbf{w}$ and $\mathbf{w} \in L^{2}\left(\Omega_{0}\right)$ with $\mathbf{w} \cdot \mathbf{k}=0$ at $z=0,1$, then in [4], one can also find

$$
\|\Pi\|_{1,2} \leq C(\beta)\|\mathbf{w}\|_{2} \text {. }
$$

If, moreover, $f=\nabla \cdot(\nabla \cdot(\mathbf{u} \otimes \mathbf{u}))$ with $\mathbf{u} \cdot \mathbf{k}=0$ at $z=$ 0,1 and $\nabla \cdot \mathbf{u}=0$, then in [4], one can also find

$$
\|\Pi\|_{2} \leq C(\beta)\|\mathbf{u}\|_{4}^{2} \text {. }
$$

Notice that both these estimates fall into the classic Navier-Stokes estimate:

$$
\|p\|_{2} \leq c\|\mathbf{v}\|_{4}^{2}
$$

as $\beta$ goes to zero.

However, here the case $\nabla \cdot \mathbf{v} \neq 0$ is, in principle, considered.

By taking the data of (29) from system (25), all hypotheses concerning the boundary conditions are verified, and by writing

$$
f=-\frac{1}{\operatorname{Pr}} \nabla \cdot(\mathbf{v} \cdot \nabla \mathbf{v}+\phi \mathbf{v})+\operatorname{Ra} \nabla \cdot(\tau \mathbf{k}),
$$

one sees that (31) can be used.

Hence,

$$
\|\nabla \Pi\|_{2} \leq C(\beta)\left(\frac{1}{\operatorname{Pr}}\left(\|\mathbf{v} \cdot \nabla \mathbf{v}\|_{2}+\|\phi \mathbf{v}\|_{2}\right)+\mathrm{Ra}\|\tau\|_{2}\right) .
$$

Now, let us introduce Galerkin's “approximate” solutions of (25). Let us denote by $\left\{\varphi_{j}\right\}_{j \in \mathbb{N}}$ and $\left\{\chi_{j}\right\}_{j \in \mathbb{N}}$ the periodic eigenfunctions of the Laplace operator, respectively, verifying Dirichlet and Neumann conditions at $\partial \Omega$, such that the vector functions $\Psi_{j}=\left(\chi_{j}, \chi_{j}, \varphi_{j}\right)$ verify the same boundary condition as $\mathbf{v}$ does: 


$$
\begin{aligned}
\Delta \Psi_{j}=-\lambda_{(j)} \Psi_{j}, & \lambda_{(j)}>0, \\
\Delta \varphi_{j}=-\eta_{(j)} \varphi_{j}, & \eta_{(j)}>0 .
\end{aligned}
$$

Moreover, $\Psi_{j}$ 's and $\varphi_{j}$ 's are complete orthogonal bases, respectively, for the vector and scalar fields in $W^{1,2}\left(\Omega_{0}\right)$. Further, they belong to $W^{2,2}\left(\Omega_{0}\right)$. The Galerkin approximation solutions $\mathbf{v}^{N}$ and $\tau^{N}$,

$$
\begin{aligned}
& \mathbf{v}^{N}=\sum_{j=1}^{N} C_{N}^{j}(t) \Psi_{j}, \\
& \tau^{N}=\sum_{j=1}^{N} A_{N}^{j}(t) \varphi_{j},
\end{aligned}
$$

are then defined in a standard way by projecting system (25) on the $\mathrm{N}$-dimensional subspace spanned by the same eigenfunctions. The ordinary differential equation system so obtained is the first order in the unknowns $C_{N}^{j}(t)$ and $A_{N}^{j}(t)$. It is an autonomous homogeneous system in a normal form, whose solutions are easily shown to exist for all $N \in \mathbb{N}$.

As a matter of fact, although the pressure terms in the momentum balance (which will be written in a compact form as $e^{-\beta z} \nabla \Pi$ ) cannot be solved a posteriori as for Navier-Stokes equations, one can nevertheless define for all $N \in \mathbb{N}$ some suitable $\Pi^{N}$ as a function of $C_{N}^{j}(t)$ and $A_{N}^{j}(t)$. To this end, let us use (34) in Lemma 2 and denote by $\mathscr{A}$ the linear operator:

$$
\begin{aligned}
\mathscr{A}\left(\Pi^{N}\right):= & \Delta \Pi^{N}-\beta \Pi_{z}^{N}=e^{\beta z} \nabla \\
& \cdot\left(-\frac{1}{\operatorname{Pr}}\left(\mathbf{v}^{N} \cdot \nabla \mathbf{v}^{N}+\phi^{N} \mathbf{v}^{N}\right)+\operatorname{Ra} \tau^{N} \mathbf{k}\right),
\end{aligned}
$$

where, of course, $\phi^{N}=\nabla \cdot \mathbf{v}^{N}$. By Lemma 2, the operator is continuously invertible for $0<\beta<2 \pi$ and

$$
\begin{aligned}
\Pi^{N}:= & -\frac{1}{\operatorname{Pr}} \sum_{k, i=1}^{N} \mathscr{A}^{-1}\left(e^{\beta z} \nabla \cdot\left(\Psi_{k} \cdot \nabla \Psi_{i}+\Psi_{i} \nabla \cdot \Psi_{k}\right)\right) C_{N}^{k}(t) C_{N}^{i}(t) \\
& +\operatorname{Ra} \mathscr{A}^{-1} \sum_{i=1}^{N}\left(e^{\beta z} \nabla \cdot\left(\varphi_{i} \mathbf{k}\right)\right) A_{N}^{i}(t),
\end{aligned}
$$

where the summation over repeated indices is understood. According to Lemma 2, the linear operator $\mathscr{A}^{-1}$ is defined in
$L^{2}\left(\Omega_{0}\right)$, and its image belongs to $W^{2,2}\left(\Omega_{0}\right)$. Moreover, $\mathscr{A}^{-1}$ does not depend on $t$.

For the sake of brevity, let us denote

$$
\begin{aligned}
\mathscr{F}_{k i}(x, y, z) & :=\mathscr{A}^{-1}\left(e^{\beta z} \nabla \cdot\left(\Psi_{k} \cdot \nabla \Psi_{i}+\Psi_{i} \nabla \cdot \Psi_{k}\right)\right), \\
\mathscr{G}_{i}(x, y, z) & :=\mathscr{A}^{-1}\left(e^{\beta z} \nabla \cdot\left(\varphi_{i} \mathbf{k}\right)\right) .
\end{aligned}
$$

Next, let us compute

$$
\begin{aligned}
\left(e^{-\beta z} \nabla \Pi^{N}, \Psi_{j}\right)= & -\frac{1}{\operatorname{Pr}} \sum_{k, i=1}^{N}\left(e^{-\beta z} \nabla \mathscr{F}_{k i}, \Psi_{j}\right) C_{N}^{k} C_{N}^{i} \\
& +\operatorname{Ra} \sum_{i=1}^{N}\left(e^{-\beta z} \nabla \mathscr{G}_{i}, \Psi_{j}\right) A_{N}^{i} .
\end{aligned}
$$

This expression can be inserted in the Galerkin system, which can be written as

$$
\begin{aligned}
\dot{C}_{N}^{j}= & -\operatorname{Pr} \lambda_{(j)} C_{N}^{j}-\sum_{k, i=1}^{N}\left(\Psi_{k} \cdot \nabla \Psi_{i}-e^{-\beta z} \nabla \mathscr{F}_{k i}, \Psi_{j}\right) C_{N}^{k} C_{N^{+}}^{i} \\
& +\operatorname{PrRa} \sum_{i=1}^{N}\left(\varphi_{i} \mathbf{k}-e^{-\beta z} \nabla \mathscr{G}_{i}, \Psi_{j}\right) A_{N}^{i},
\end{aligned}
$$

$\dot{A}_{N}^{j}=-\sum_{k, i=1}^{N} C_{N}^{k} A_{N}^{i}\left(\Psi_{k} \cdot \nabla \varphi_{i}, \varphi_{j}\right)-\eta_{(j)} A_{N}^{j}+C_{N}^{j}$.

It allows for solutions since the right-hand side is a Lipschitz continuous function of the variables (in fact, it is at most quadratic). For all $N \in \mathbb{N}$, the initial conditions $C_{N}^{j}(0)=C_{0}^{j}=\left(\mathbf{v}_{0}, \Psi_{j}\right)$ and $A_{N}^{j}(0)=A_{0}^{j}=\left(\tau_{0}, \Psi_{j}\right)$ correspond to the initial conditions of (25). Since the aim is still to derive the equivalence of the differential systems (17) and (19) by showing existence with $\phi=0$, the required regularity for the initial data is $W^{2,2}$. In particular, one has to define the space as the closure of linear combinations of the eigenfunctions $\chi_{j}-\left\langle\chi_{j}\right\rangle$ so that $v^{x}, v^{y} \in \mathscr{W}^{2,2}\left(\Omega_{0}\right)$, while $v^{z}, \tau \in W_{0}^{2,2}\left(\Omega_{0}\right)$, which is directly obtained by closing the $\varphi_{j}$ 's.

Moreover, an estimate for $\left\|\mathbf{v}_{t}^{N}(0)\right\|_{2}$ and $\left\|\tau_{t}^{N}(0)\right\|_{2}$ is hereafter needed, for which the left-hand side of (42) evaluated in $t=0$ has to be increased by directly inserting (34) in estimate (31):

$$
\begin{aligned}
\left|\left(e^{-\beta z} \nabla \Pi^{N}, \Psi_{j}\right)\right| & \leq e^{-\beta}\left|\left(\nabla \Pi^{N}, \Psi_{j}\right)\right| \\
& \leq C(\beta, \operatorname{Pr}, \operatorname{Ra})\left(\left|\left(\mathbf{v}^{N} \cdot \nabla \mathbf{v}^{N}+\phi^{N} \mathbf{v}^{N}, \Psi_{j}\right)\right|+\left|\left(\tau^{N}, \Psi_{j}\right)\right|\right) \\
& \leq C(\beta, \operatorname{Pr}, \operatorname{Ra})\left(\sum_{k, i=1}^{N}\left|C_{N}^{k} C_{N}^{i}\left(\Psi_{k} \cdot \nabla \Psi_{i}+\Psi_{i} \nabla \cdot \Psi_{k}, \Psi_{j}\right)\right|+\left|A_{N}^{j}\right|\right) .
\end{aligned}
$$


After squaring and summing the inequalities derived by (42) and evaluating at the initial time, one obtains a bound independent of $N$, estimated by the $W^{2,2}$-norm of the initial data through Bessel's inequality. Since the basis is in $W^{2,2}\left(\Omega_{0}\right)$, by linearity

$$
\left\|\Delta \mathbf{v}^{N}(0)\right\|_{2}=\left\|\sum_{j=1}^{N} \lambda_{(j)}\left(\mathbf{v}_{0}, \Psi_{j}\right) \Psi_{j}\right\|_{2}=\left\|\sum_{j=1}^{N}\left(\mathbf{v}_{0}, \Delta \Psi_{j}\right) \Psi_{j}\right\|_{2}=\left\|\sum_{j=1}^{N}\left(\Delta \mathbf{v}_{0}, \Psi_{j}\right) \Psi_{j}\right\|_{2} \leq\left\|\Delta \mathbf{v}_{0}\right\|_{2} .
$$

Concerning the nonlinear terms, one can compute, for instance,

$$
\begin{aligned}
& \sum_{j=1}^{N} \sum_{k, i=1}^{N} \sum_{l, n=1}^{N}\left(\Psi_{k} \cdot \nabla \Psi_{i}, \Psi_{j}\right)\left(\Psi_{l} \cdot \nabla \Psi_{n}, \Psi_{j}\right) C_{N}^{k}(0) C_{N}^{i}(0) C_{N}^{l}(0) C_{N}^{n}(0) \\
& \quad \leq \sum_{j=1}^{\infty}\left(\mathbf{v}_{0} \cdot \nabla \mathbf{v}_{0}, \Psi_{j}\right)^{2}=\left\|\mathbf{v}_{0} \cdot \nabla \mathbf{v}_{0}\right\|_{2} .
\end{aligned}
$$

Next, these nonlinear terms at the right-hand side can be increased by (12) and (13), through Hölder $(p=(3 / 2)$ and $\left.p^{\prime}=3\right)$ and interpolation $\left(p=(4 / 3)\right.$ and $\left.p^{\prime}=4\right)$ inequalities (the procedure is detailed in the proof of Lemma 3 ). Finally, using the Poincaré inequality and (10), one gets

$$
\begin{aligned}
& \left\|\mathbf{v}_{t}^{N}(0)\right\|_{2} \leq C(\beta, \operatorname{Pr}, \operatorname{Ra})\left(\left\|\Delta \mathbf{v}_{0}\right\|_{2}+\left\|\tau_{0}\right\|_{2}+\left\|\Delta \mathbf{v}_{0}\right\|_{2}^{2}\right), \\
& \left\|\tau_{t}^{N}(0)\right\|_{2} \leq C(\beta, \operatorname{Pr}, \operatorname{Ra})\left(\left\|\Delta \tau_{0}\right\|_{2}+\left\|\mathbf{v}_{0}\right\|_{2}+\left\|\nabla \mathbf{v}_{0}\right\|_{2}\left\|\Delta \tau_{0}\right\|_{2}\right) .
\end{aligned}
$$

Here and hereafter, the $L^{2}$-norms of the two nonlinear terms $\mathbf{v} \cdot \nabla \mathbf{v}$ and $\phi \mathbf{v}$ are estimated exactly in the same way, but for brevity only the first one will be developed.

\section{Evolutive Estimates and Main Results}

Let us formally derive the $a$ priori estimates for solutions of system (25): by quite a standard procedure, they are verified just by Galerkin solutions, denoted here by $(\mathbf{v}, \tau)$, and, as seen, are bound uniformly with respect to $N$.

Lemma 3. Let us define the energy functions:

$$
\begin{aligned}
& E_{1}(t):=\frac{1}{2}\left(\frac{\|\mathbf{v}\|_{2}^{2}}{\operatorname{Pr}}+\operatorname{Ra}\|\tau\|_{2}^{2}\right), \\
& E_{2}(t):=\frac{1}{2}\left(\frac{\|\nabla \mathbf{v}\|_{2}^{2}}{\operatorname{Pr}}+\operatorname{Ra}\|\nabla \tau\|_{2}^{2}\right) .
\end{aligned}
$$

Then, for sufficiently small $\varepsilon>0$, the following inequalities hold true:

$$
\begin{aligned}
& \frac{\mathrm{d} E_{1}}{\mathrm{~d} t}+c\left(\|\nabla \mathbf{v}\|_{2}^{2}+\operatorname{Ra}\|\nabla \tau\|_{2}^{2}\right) \leq C(\beta, \operatorname{Pr}, \operatorname{Ra})\left(E_{1}^{3}+E_{2}^{3 / 2}+\varepsilon\|\Delta \mathbf{v}\|_{2}^{2}\right)+C(\beta, \operatorname{Pr}) \frac{\mathrm{Ra}}{\varepsilon}\|\mathbf{v}\|_{2}^{2}, \\
& \frac{\mathrm{d} E_{2}}{\mathrm{~d} t}+c\left(\|\Delta \mathbf{v}\|_{2}^{2}+\operatorname{Ra}\|\Delta \tau\|_{2}^{2}\right) \leq C(\beta, \operatorname{Pr}, \operatorname{Ra}) E_{2}^{3}+C(\beta, \operatorname{Pr}) \frac{\mathrm{Ra}}{\varepsilon} E_{1}, \\
& \frac{\mathrm{d} E_{2}}{\mathrm{~d} t}+c\left(\|\Delta \mathbf{v}\|_{2}^{2}+\operatorname{Ra}\|\tau\|_{2}^{2}\right) \leq C(\beta, \operatorname{Pr}, \operatorname{Ra}) E_{2}^{3}+C(\beta, \operatorname{Pr}) \frac{\mathrm{Ra}}{\varepsilon}\|\Delta \mathbf{v}\|_{2}^{2},
\end{aligned}
$$

where $c \in(0,1)$.

Proof. One can test (25) with (v, $\tau$ ), and then one multiplies the third equation by Ra and then sums:

$$
\begin{aligned}
\frac{\mathrm{d}}{\mathrm{d} t} E_{1}(t)+\|\nabla \mathbf{v}\|_{2}^{2}+\operatorname{Ra}\|\nabla \tau\|_{2}^{2}= & -\left(e^{-\beta z} \nabla \Pi, \mathbf{v}\right)+2 \operatorname{Ra}\left(\tau, v^{z}\right) \\
& -\frac{1}{\operatorname{Pr}}(\mathbf{v} \cdot \nabla \mathbf{v}, \mathbf{v})-\frac{1}{\operatorname{Pr}}(\phi \mathbf{v}, \mathbf{v})-\operatorname{Ra}(\mathbf{v} \cdot \nabla \tau, \tau)
\end{aligned}
$$


In order to estimate the nonlinear term arising from the coupling with the pressure equation, one uses (35): the nonlinear term at the right-hand side can be increased by
(12) and (13), through Hölder $\left(p=(3 / 2)\right.$ and $\left.p^{\prime}=3\right)$ and interpolation $\left(p=(4 / 3)\right.$ and $\left.p^{\prime}=4\right)$ inequalities, so that

$$
\begin{aligned}
\left.\left(e^{-\beta z} \nabla \Pi, \mathbf{v}\right)\right|_{\tau=0} & \leq c\|\mathbf{v} \cdot \nabla \mathbf{v}\|_{2}\|\mathbf{v}\|_{2} \leq c\|\mathbf{v}\|_{3}\|\nabla \mathbf{v}\|_{6}\|\mathbf{v}\|_{2} \leq c\|\mathbf{v}\|_{2}^{1 / 2}\|\mathbf{v}\|_{6}^{1 / 2}\|\Delta \mathbf{v}\|_{2}\|\mathbf{v}\|_{2} \\
& \leq c\|\mathbf{v}\|_{2}^{3 / 2}\|\nabla \mathbf{v}\|_{2}^{1 / 2}\|\Delta \mathbf{v}\|_{2} \leq \frac{c}{4 \varepsilon^{2}}\|\mathbf{v}\|_{2}^{6}+\frac{c \varepsilon}{4}\|\nabla \mathbf{v}\|_{2}^{2}+\frac{c \varepsilon}{2}\|\Delta \mathbf{v}\|_{2}^{2} .
\end{aligned}
$$

This term is multiplied by $\beta(C(\beta) / \mathrm{Pr})$, while the linear part of the pressure estimate can be included in the estimate of the buoyancy term and of the energy equation transport term, giving rise (in the whole) to

$$
\operatorname{RaC}(\beta, \operatorname{Pr})\|\tau\|_{2}\|\mathbf{v}\|_{2} \leq \operatorname{RaC}(\beta, \operatorname{Pr})\left(\frac{\varepsilon}{2}\|\tau\|_{2}^{2}+\frac{1}{2 \varepsilon}\|\mathbf{v}\|_{2}^{2}\right) .
$$

Here, the term with the second derivatives does not allow to get an autonomous bound for $E_{1}(t)$, so that a further estimate for higher derivatives need to be added. Notice that the convective terms do not vanish, and by also resorting to Young's inequality, they are bounded as follows:

$$
\begin{aligned}
(\mathbf{v} \cdot \nabla \mathbf{v}, \mathbf{v}) & =\int_{\Omega_{0}} \nabla \cdot\left(\mathbf{v} \frac{v^{2}}{2}\right)-\int_{\Omega_{0}} \phi \frac{v^{2}}{2} \leq \frac{1}{2}\|\phi\|_{2}\|\mathbf{v}\|_{4}^{2} \\
& \leq C\|\nabla \mathbf{v}\|_{2}\|\mathbf{v}\|_{2}^{1 / 2}\|\mathbf{v}\|_{6}^{3 / 2} \leq C\|\nabla \mathbf{v}\|_{2}^{3}, \\
(\mathbf{v} \cdot \nabla \tau, \tau) & =\int_{\Omega_{0}} \nabla \cdot\left(\mathbf{v} \frac{\tau^{2}}{2}\right)-\int_{\Omega_{0}} \phi \frac{\tau^{2}}{2} \leq \frac{1}{2}\|\phi\|_{2}\|\tau\|_{4}^{2} \\
& \leq C\|\nabla \mathbf{v}\|_{2}\|\tau\|_{2}^{1 / 2}\|\tau\|_{6}^{3 / 2} \leq C\|\nabla \mathbf{v}\|_{2}\|\nabla \tau\|_{2}^{2} \leq \frac{1}{3} C\|\nabla \mathbf{v}\|_{2}^{3}+\frac{2}{3} C\|\nabla \tau\|_{2}^{3} .
\end{aligned}
$$

Analogously,

$$
\int_{\Omega_{0}} \phi v^{2} \leq\|\nabla \mathbf{v}\|_{2}^{3} .
$$

By collecting all these estimates, using the Poincare inequality, increasing $\|\nabla \mathbf{v}\|_{2}^{3}$ with $E_{2}^{3 / 2}$, and absorbing at the left-hand side, where possible, the terms of order $\epsilon$, the first inequality follows.

Now, the balance equations are tested with $\Delta \mathbf{v}$ and $\Delta \tau$, respectively, and again (35) is inserted.

First, the following equations are written:

$$
\begin{aligned}
\frac{1}{2 \operatorname{Pr}} \frac{\mathrm{d}}{\mathrm{d} t}\|\nabla \mathbf{v}\|_{2}^{2}+\|\Delta \mathbf{v}\|_{2}^{2}= & \frac{1}{\operatorname{Pr}}(\mathbf{v} \cdot \nabla \mathbf{v}+\phi \mathbf{v}, \Delta \mathbf{v})+\left(e^{-\beta z} \nabla \Pi, \Delta \mathbf{v}\right)-\operatorname{Ra}(\tau \mathbf{k}, \Delta \mathbf{v}), \\
\frac{1}{2} \frac{\mathrm{d}}{\mathrm{d} t}\|\nabla \tau\|_{2}^{2}+\|\Delta \tau\|_{2}^{2}= & (\mathbf{v} \cdot \nabla \tau, \Delta \tau)+\left(v^{z}, \Delta \tau\right), \\
\frac{\mathrm{d} E_{2}}{\mathrm{~d} t}+\|\Delta \mathbf{v}\|_{2}^{2}+\operatorname{Ra}\|\Delta \tau\|_{2}^{2}= & \left(e^{-\beta z} \nabla \Pi, \Delta \mathbf{v}\right)+\operatorname{Ra}\left(\tau, \Delta v^{z}\right)+\operatorname{Ra}\left(\Delta \tau, v^{z}\right) \\
& \frac{1}{\operatorname{Pr}}(\mathbf{v} \cdot \nabla \mathbf{v}, \Delta \mathbf{v})+\frac{1}{\operatorname{Pr}}(\phi \mathbf{v}, \Delta \mathbf{v})+\operatorname{Ra}(\mathbf{v} \cdot \nabla \tau, \Delta \tau) .
\end{aligned}
$$

Again, the pressure-related convective terms are bounded, and then the temperature-depending coupling terms are estimated:

$$
\begin{array}{r}
\left.\left(e^{-\beta z} \nabla \Pi, \Delta \mathbf{v}\right)\right|_{\tau=0} \leq c\|\mathbf{v} \cdot \nabla \mathbf{v}\|_{2}\|\Delta \mathbf{v}\|_{2} \leq\|\mathbf{v}\|_{6}\|\nabla \mathbf{v}\|_{3}\|\Delta \mathbf{v}\|_{2} \leq \\
c\|\mathbf{v}\|_{6}\|\nabla \mathbf{v}\|_{2}^{1 / 2}\left\|D^{2} \mathbf{v}\right\|_{2}^{1 / 2}\|\Delta \mathbf{v}\|_{2} \leq c\|\nabla \mathbf{v}\|_{2}^{3 / 2}\|\Delta \mathbf{v}\|_{2}^{3 / 2} \leq \frac{3 c \varepsilon}{4}\|\Delta \mathbf{v}\|_{2}^{2}+\frac{c}{4 \varepsilon}\|\nabla \mathbf{v}\|_{2}^{6} .
\end{array}
$$


In order to reach local-in-time existence of solutions for any initial data, one writes

$$
\operatorname{RaC}(\beta, \operatorname{Pr})\left(\|\tau\|_{2}\|\Delta \mathbf{v}\|_{2}+\|\Delta \tau\|_{2}\|\mathbf{v}\|_{2}\right) \leq \operatorname{RaC}(\beta, \operatorname{Pr})\left(\frac{\varepsilon}{2}\|\Delta \tau\|_{2}^{2}+\frac{\varepsilon}{2}\|\Delta \mathbf{v}\|_{2}^{2}+\frac{1}{2 \varepsilon^{2}} E_{1}(t)\right)
$$

In order to achieve global-in-time existence for small Now, the nonlinear terms are focused: initial data, one uses an alternative estimate, which follows by several applications of the Poincaré inequality:

$$
\begin{gathered}
\operatorname{RaC}(\beta, \operatorname{Pr})\left(\|\tau\|_{2}\|\Delta \mathbf{v}\|_{2}+\|\Delta \tau\|_{2}\|\mathbf{v}\|_{2}\right) \\
\leq \operatorname{RaC}(\beta, \operatorname{Pr})\left(\frac{\varepsilon}{2}\|\Delta \tau\|_{2}^{2}+\frac{1}{2 \varepsilon}\|\Delta \mathbf{v}\|_{2}^{2}\right) .
\end{gathered}
$$

$$
\begin{aligned}
(\mathbf{v} \cdot \nabla \mathbf{v}+\phi \mathbf{v}, \Delta \mathbf{v}) & \leq C\|\mathbf{v}\|_{6}\|\nabla \mathbf{v}\|_{3}\|\Delta \mathbf{v}\|_{2} \leq C\|\mathbf{v}\|_{6}\|\nabla \mathbf{v}\|_{2}^{1 / 2}\|\nabla \mathbf{v}\|_{6}^{1 / 2}\|\Delta \mathbf{v}\|_{2} \\
& \leq C\|\nabla \mathbf{v}\|_{2}^{3 / 2}\|\Delta \mathbf{v}\|_{2}^{3 / 2} \leq \frac{3 \varepsilon}{4} C\|\Delta \mathbf{v}\|_{2}^{2}+\frac{1}{4 \varepsilon} C\|\nabla \mathbf{v}\|_{2}^{6}, \\
(\mathbf{v} \cdot \nabla \tau, \Delta \tau) & \leq\|\mathbf{v}\|_{6}\|\nabla \tau\|_{3}\|\Delta \tau\|_{2} \leq C\|\nabla \mathbf{v}\|_{2}\|\nabla \tau\|_{2}^{1 / 2}\left\|D^{2} \tau\right\|_{2}^{1 / 2}\|\Delta \tau\|_{2} \\
& \leq C\|\nabla \mathbf{v}\|_{2}\|\nabla \tau\|_{2}^{1 / 2}\|\Delta \tau\|_{2}^{3 / 2} \leq \frac{3 \varepsilon}{4} C\|\Delta \tau\|_{2}^{2}+\frac{1}{4 \varepsilon} C\|\nabla \tau\|_{2}^{2}\|\nabla \mathbf{v}\|_{2}^{4} \\
& \leq \frac{3 \varepsilon}{4} C\|\Delta \tau\|_{2}^{2}+\frac{1}{12 \varepsilon} C\|\nabla \tau\|_{2}^{6}+\frac{1}{6 \varepsilon} C\|\nabla \mathbf{v}\|_{2}^{6} .
\end{aligned}
$$

The terms of order $\epsilon$ are still absorbed at the left-hand side, and then inequalities (50) and (51), respectively, follow by (59) and (60).

Lemma 4. Let

$$
T:=\log \left(\frac{E^{2}(0)+1}{E^{2}(0)}\right)^{1 / 4 C}
$$

then for all $t \in(0, T]$, the following estimates hold:

$$
E(t) \leq\left(E(0) \frac{\sqrt{1+E^{2}(0)}}{1+E(0)\left(E(0)-\sqrt{1+E^{2}(0)}\right)}\right)^{1 / 2},
$$

where $E(t):=E_{1}(t)+E_{2}(t)$. Furthermore,

$$
\int_{0}^{T} \mathscr{P}(t) \mathrm{d} t \leq C \log \left(\frac{E^{2}(0)+1}{E^{2}(0)}\right)^{1 / 4}\left(E(0)+E^{1 / 2}(0)\right),
$$

where $\mathscr{P}(t):=\|\nabla \mathbf{v}(t)\|_{2}^{2}+\|\Delta \mathbf{v}(t)\|_{2}^{2}+\|\nabla \tau(t)\|_{2}^{2}+\|\Delta \tau(t)\|_{2}^{2}$.

Proof. By summing side-by-side the first and second inequalities in Lemma 3, one obtains

$$
\frac{\mathrm{d}}{\mathrm{d} t} E(t)+c_{1} \mathscr{P}(t) \leq c_{2}\left(E(t)+E^{3 / 2}(t)+E^{3}(t)\right) .
$$

Here, one has

$$
E^{3 / 2}=E^{3 / 4} E^{3 / 4} \leq \frac{3}{4} E+\frac{1}{4} E^{3},
$$

and consequently the right-hand side can be bounded by $E+E^{3}$. Hence,

$$
\int_{E(0)}^{E(t)} \frac{\mathrm{d} E}{E\left(E^{2}+1\right)}=\int_{E(0)}^{E(t)}\left(\frac{1}{E}-\frac{E}{E^{2}+1}\right) \mathrm{d} E \leq C t,
$$

then

$$
\frac{E(t) \sqrt{E^{2}(0)+1}}{E(0) \sqrt{E^{2}(t)+1}} \leq e^{C t}
$$

It follows that once the maximal interval corresponding to a vanishing denominator is identified,

$$
\forall t \in(0, T) \text { with } 2 T:=\log \left(\frac{E^{2}(0)+1}{E^{2}(0)}\right)^{1 / 2 C},
$$

$$
\begin{aligned}
E^{2}(t) & \leq \frac{e^{2 C t} E^{2}(0)}{1+E^{2}(0)\left(1-e^{2 C t}\right)} \\
& \leq E(0) \frac{\sqrt{1+E^{2}(0)}}{1+E(0)\left(E(0)-\sqrt{1+E^{2}(0)}\right)},
\end{aligned}
$$


the energy is bounded.

Next, coming back to (65) and integrating in $[0, T)$, one deduces a bound for the norm in $L^{\infty}\left(0, T ; W^{1,2}\left(\Omega_{0}\right)\right) \cap$ $L^{2}\left(0, T ; W^{2,2}\left(\Omega_{0}\right)\right)$, as can be seen hereafter: the inequality

$$
\int_{0}^{T} \mathscr{P}(t) \mathrm{d} t \leq C \log \left(\frac{E^{2}(0)+1}{E^{2}(0)}\right)^{1 / 4}\left(E(0)+E^{1 / 2}(0)\right),
$$

implies in particular the integrability of $\|\nabla \mathbf{v}(t)\|_{2}^{2}$ and of $\|\Delta \mathbf{v}(t)\|_{2}^{2}$.

Although, the Galerkin solutions exist for all $t>0$, their uniform estimates exist only in the finite interval defined in (69). Global-in-time solution will be found only by asking suitable smallness of Ra and of the initial data.

Finally, (25) is derived formally with respect to $t$, and the same procedure is repeated as in the first estimate, but for the nonlinear convective terms. Now, in order to bound the initial data for $\mathbf{v}_{t}$, initial data in $W^{2,2}$ is chosen:

$$
\left\{\begin{array}{l}
\Delta \Pi_{t}-\beta \Pi_{t z}=-\frac{e^{\beta z}}{\operatorname{Pr}} \nabla \cdot\left(\mathbf{v}_{t} \cdot \nabla \mathbf{v}+\phi_{t} \mathbf{v}+\mathbf{v} \cdot \nabla \mathbf{v}_{t}+\phi \mathbf{v}_{t}\right) \\
\quad+\operatorname{Ra} e^{\beta z} \tau_{t z}, \\
\frac{1}{\operatorname{Pr}}\left(\frac{\partial \mathbf{v}_{t}}{\partial t}+\mathbf{v}_{t} \cdot \nabla \mathbf{v}+\phi_{t} \mathbf{v}+\mathbf{v} \cdot \nabla \mathbf{v}_{t}+\phi \mathbf{v}_{t}\right)-\Delta \mathbf{v}_{t} \\
\quad=-\nabla\left(e^{-\beta z} \Pi_{t}\right)-\beta e^{-\beta z} \Pi_{t} \mathbf{k}+\operatorname{Ra} \tau_{t} \mathbf{k} \\
\frac{\partial \tau_{t}}{\partial t}+\mathbf{v}_{t} \cdot \nabla \tau+\mathbf{v} \cdot \nabla \tau_{t}-\Delta \tau_{t}=v_{t}^{z}
\end{array}\right.
$$

Again, the terms depending on $\phi$ or $\phi_{t}$ will be bounded exactly as the corresponding convective ones.

Lemma 5. Let us define the energy function as

$$
\mathscr{E}(t):=\frac{1}{2}\left(\frac{\left\|\mathbf{v}_{t}\right\|_{2}^{2}}{\operatorname{Pr}}+\operatorname{Ra}\left\|\tau_{t}\right\|_{2}^{2}\right) .
$$

Then, for sufficiently small $\varepsilon>0$, the following inequality holds true:

$$
\begin{aligned}
\frac{\mathrm{d} \mathscr{E}}{\mathrm{d} t}(t)+c\left(\left\|\nabla \mathbf{v}_{t}\right\|_{2}^{2}+\operatorname{Ra}\left\|\nabla \tau_{t}\right\|_{2}^{2}\right) \leq & C(\beta, \operatorname{Pr}, \operatorname{Ra})\|\Delta \mathbf{v}\|_{2}^{2} \mathscr{E}(t) \\
& +C(\beta, \operatorname{Pr}) \frac{\operatorname{Ra}}{\varepsilon}\left\|\mathbf{v}_{t}\right\|_{2}^{2},
\end{aligned}
$$

where $c \in(0,1)$.

Proof. Let us start with the equation

$$
\begin{aligned}
\frac{\mathrm{d}}{\mathrm{d} t} \mathscr{E}(t)+\left\|\nabla \mathbf{v}_{t}\right\|_{2}^{2}+\operatorname{Ra}\left\|\nabla \tau_{t}\right\|_{2}^{2}= & -\left(e^{-\beta z} \nabla \Pi_{t}, \mathbf{v}_{t}\right)+2 \operatorname{Ra}\left(\tau_{t}, v_{t}^{z}\right)+ \\
& -\frac{1}{\operatorname{Pr}}\left(\mathbf{v}_{t} \cdot \nabla \mathbf{v}+\mathbf{v} \cdot \nabla \mathbf{v}_{t}, \mathbf{v}_{t}\right)-\frac{1}{\operatorname{Pr}}\left(\phi_{t} \mathbf{v}+\phi \mathbf{v}_{t}, \mathbf{v}_{t}\right)-\operatorname{Ra}\left(\mathbf{v}_{t} \cdot \nabla \tau+\mathbf{v} \cdot \nabla \tau_{t}, \tau_{t}\right) .
\end{aligned}
$$

Technically, the estimates of the nonlinear convective terms are the only different ones from the previous ones, since the terms are not homogeneous:

$$
\begin{aligned}
\left.\left(e^{-\beta z} \nabla \Pi_{t}, \mathbf{v}_{t}\right)\right|_{\tau_{t}=0} & \leq c\left\|\mathbf{v}_{t}\right\|_{2}\left(\left\|\mathbf{v}_{t} \cdot \nabla \mathbf{v}\right\|_{2}+\left\|\mathbf{v} \cdot \nabla \mathbf{v}_{t}\right\|_{2}\right) \\
& \leq c\left(\left\|\mathbf{v}_{t}\right\|_{2}\left\|\mathbf{v}_{t}\right\|_{3}\|\nabla \mathbf{v}\|_{6}+\left\|\mathbf{v}_{t}\right\|\left\|_{2}\right\| \mathbf{v}\left\|_{\infty}\right\| \nabla \mathbf{v}_{t} \|_{2}\right) \\
& \leq c\left(\left\|\mathbf{v}_{t}\right\|_{2}^{3 / 2}\left\|\nabla \mathbf{v}_{t}\right\|_{2}^{1 / 2}\|\Delta \mathbf{v}\|_{2}+\left\|\mathbf{v}_{t}\right\|\|\Delta \mathbf{v}\|_{2}\left\|\nabla \mathbf{v}_{t}\right\|_{2}\right) \\
& \leq c\left(\|\Delta \mathbf{v}\|_{2}^{2}\left\|\mathbf{v}_{t}\right\|_{2}^{2}+\varepsilon\left\|\nabla \mathbf{v}_{t}\right\|_{2}^{2}\right) .
\end{aligned}
$$

The same estimates can be repeated for the other nonlinear terms, such as

$$
\begin{aligned}
\left(\phi \mathbf{v}_{t}, \mathbf{v}_{t}\right) & \leq c\|\nabla \mathbf{v}\|_{6}\left\|\mathbf{v}_{t}\right\|_{2}\left\|\mathbf{v}_{t}\right\|_{3}, \\
\left(\phi_{t} \mathbf{v}, \mathbf{v}_{t}\right) & \leq c\left\|\mathbf{v}_{t}\right\|_{2}\|\mathbf{v}\|_{\infty}\left\|\nabla \mathbf{v}_{t}\right\|_{2}, \\
\left(\mathbf{v}_{t} \cdot \nabla \tau, \tau_{t}\right) & \leq c\|\nabla \tau\|_{6}\left\|\mathbf{v}_{t}\right\|_{2}\left\|\tau_{t}\right\|_{3}, \\
\left(\mathbf{v} \cdot \nabla \tau_{t}, \tau_{t}\right) & \leq c\left\|\tau_{t}\right\|_{2}\|\mathbf{v}\|_{\infty}\left\|\nabla \tau_{t}\right\|_{2} .
\end{aligned}
$$
side

The gradient terms can be absorbed at the left-hand

Starting from (74), where the positive term at the lefthand side can be neglected and the term $\left\|\mathbf{v}_{t}\right\|_{2}^{2}$ at the right- 
hand side can be bounded by $\mathscr{E}(t)$, by a generalized Gronwall argument, one shows that

$$
\mathscr{E}(t) \leq e^{c \int_{0}^{T}\left(\|\Delta \mathbf{v}(t)\|_{2}^{2}+1\right) \mathrm{d} t} \mathscr{E}(0) .
$$

As a matter of fact, $(71)$ implies $\mathbf{v} \in L^{2}\left(0, T ; W^{2,2}\left(\Omega_{0}\right)\right)$, so that $\mathscr{E}(t)$ is bounded in $(0, T)$. Further, by coming back to (74) and integrating again, it follows also

$$
\int_{0}^{T}\left(\left\|\nabla \mathbf{v}_{t}\right\|_{2}^{2}+\left\|\nabla \tau_{t}\right\|_{2}^{2}\right) \mathrm{d} t \leq O\left(\left\|\mathbf{v}_{0}\right\|_{2,2},\left\|\tau_{0}\right\|_{2,2}\right),
$$

and this is independent of the size of Ra.

Theorem 1. Let $\beta<2 \pi$ and $\operatorname{Pr}$ and $R a$ be arbitrary, then system (25) with initial data $\left(\mathbf{v}_{0}, \tau_{0}\right) \in W^{2,2}\left(\Omega_{0}\right)$ admits a solution a la Ladyzhenskaya, namely,

$$
\begin{aligned}
(\mathbf{v}, \tau) & \in L^{\infty}\left(0, T ; W^{2,2}\left(\Omega_{0}\right)\right) \cap L^{2}\left(0, T ; W^{3,2}\left(\Omega_{0}\right)\right), \\
\left(\mathbf{v}_{t}, \tau_{t}\right) & \in L^{\infty}\left(0, T ; L^{2}\left(\Omega_{0}\right)\right) \cap L^{2}\left(0, T ; W^{1,2}\left(\Omega_{0}\right)\right),
\end{aligned}
$$

where

$$
T:=\log \left(\frac{E^{2}(0)+1}{E^{2}(0)}\right)^{1 / 4 C} .
$$

Moreover, if $\nabla \cdot \mathbf{v}_{0}=0$, then $\nabla \cdot \mathbf{v}(t)=0$ for all $t \in(0, T]$. Finally, if $R a,\left\|\mathbf{v}_{0}\right\|_{2,2}$, and $\left\|\tau_{0}\right\|_{2,2}$ are sufficiently small, depending on $\operatorname{Pr}$ and $\beta$, then $T=\infty$ is taken in (80) and $(\mathbf{v}, \tau)$ decays exponentially fast to zero.

Proof. Employing the estimates proved in Lemma 4, it is routine to show that the approximate solutions constructed with the Galerkin method converge to a solution:

$$
\begin{aligned}
(\mathbf{v}, \tau) & \in L^{\infty}\left(0, T ; W^{1,2}\left(\Omega_{0}\right)\right) \cap L^{2}\left(0, T ; W^{2,2}\left(\Omega_{0}\right)\right), \\
\Pi & \in L^{2}\left(0, T ; W^{2,2}\left(\Omega_{0}\right)\right), \\
\left(\mathbf{v}_{t}, \tau_{t}\right) & \in L^{\infty}\left(0, T ; L^{2}\left(\Omega_{0}\right)\right) \cap L^{2}\left(0, T ; W^{1,2}\left(\Omega_{0}\right)\right),
\end{aligned}
$$

where $T$ is given in (81).

The proof of the further regularity properties, implying that the solution fulfils system (17) too, is based on (78) and (79) and in particular on the uniform boundedness of $\left\|\mathbf{v}_{t}\right\|_{2}$, as well as the integrability of $\left\|\nabla \mathbf{v}_{t}(t)\right\|_{2}^{2}$. To this end, viewing the momentum balance as a Poisson problem and increasing the pressure in the usual way, the following is obtained with the help of Lemma 1:

$$
\begin{aligned}
\|\mathbf{v}(t)\|_{2,2} & \leq C\left(\|\mathbf{v} \cdot \nabla \mathbf{v}\|_{2}+\|\nabla \Pi\|_{2}+\left\|\mathbf{v}_{t}\right\|_{2}+\|\tau\|_{2}\right) \\
& \leq C\left(\|\nabla \mathbf{v}\|_{2}^{4}+\varepsilon\|\Delta \mathbf{v}\|_{2}^{2}+\left\|\mathbf{v}_{t}\right\|_{2}+\|\tau\|_{2}\right) .
\end{aligned}
$$

The $\varepsilon$ term at the right-hand side can be absorbed by the left-hand side, so that finally the following bound is obtained:

$$
\begin{aligned}
\text { ess } \sup _{(0, T)}\|\mathbf{v}(t)\|_{2,2} & \leq \text { Cess } \sup _{(0, T)}\left(\|\nabla \mathbf{v}(t)\|_{2}^{4}+\|\tau\|_{2}+\left\|\mathbf{v}_{t}\right\|_{2}\right) \\
& \leq O\left(\left\|\mathbf{v}_{0}\right\|_{2,2},\left\|\tau_{0}\right\|_{2,2}\right) .
\end{aligned}
$$

Moreover, again from Lemma 1, it is also obtained that

$$
\int_{0}^{T}\|\mathbf{v}(t)\|_{3,2}^{2} \mathrm{~d} t \leq C \int_{0}^{T}\left(\|\mathbf{v} \cdot \nabla \mathbf{v}\|_{1,2}^{2}+\|\Pi\|_{2,2}^{2}+\left\|\mathbf{v}_{t}\right\|_{1,2}^{2}+\|\tau\|_{1,2}^{2}\right) \mathrm{d} t .
$$

It is remarked that, as noticed in Section 2, the compatibility condition related to the Neumann problem is verified. Here, the nonlinear terms at the right-hand side can be increased by using (14) and interpolation inequality:

$$
\begin{aligned}
& \left\|\mathbf{v} \cdot D^{2} \mathbf{v}\right\|_{2} \leq c\|\mathbf{v}\|_{\infty}\left\|D^{2} \mathbf{v}\right\|_{2} \leq c\left\|D^{2} \mathbf{v}\right\|_{2}^{2}, \\
& \left\|\nabla \mathbf{v}(\nabla \mathbf{v})^{T}\right\| \leq c\|\nabla \mathbf{v}\|_{4}^{2} \leq c\|\nabla \mathbf{v}\|_{6}\|\nabla \mathbf{v}\|_{3} \leq c\left\|D^{2} \mathbf{v}\right\|_{2}^{2} .
\end{aligned}
$$

The proof that $\tau$ is bounded in $L^{\infty}\left(0, T ; W^{1,2}\left(\Omega_{0}\right)\right) \cap$ $L^{2}\left(0, T ; W^{2,2}\left(\Omega_{0}\right)\right)$ is similar and simpler.

The proof of global existence follows from Lemmas 3 and 5 by setting

$$
\begin{aligned}
E_{0}(t):= & E(t)+\mathscr{E}(t), \\
\mathscr{P}_{0}(t):= & \|\nabla \mathbf{v}(t)\|_{2}^{2}+\|\Delta \mathbf{v}(t)\|_{2}^{2}+\left\|\nabla \mathbf{v}_{t}(t)\right\|_{2}^{2} \\
& +\operatorname{Ra}\left(\|\nabla \tau(t)\|_{2}^{2}+\|\Delta \tau(t)\|_{2}^{2}+\left\|\nabla \tau_{t}(t)\right\|_{2}^{2}\right) .
\end{aligned}
$$

In fact, if inequalities (49), (51), and (74) are added, bringing to the left-hand side the three terms with factor $\mathrm{Ra} / \varepsilon$ and using the Poincaré inequality

$$
E \leq k \mathscr{P},
$$

to absorb them in $\mathscr{P}_{0}$ for sufficiently small $\mathrm{Ra}$ (depending on $\varepsilon$ ), one gets

$$
\frac{\mathrm{d}}{\mathrm{d} t} E(t)+c_{1} \mathscr{P}(t) \leq c_{2}\left(E^{3 / 2}(t)+E^{3}(t)\right) .
$$

The constant $c_{1}$ is now fixed positive by suitably restricting Ra. Again, using the Poincaré inequality on the left-hand side of (89), one infers

$$
\frac{\mathrm{d}}{\mathrm{d} t} E(t)+\left[c_{1}-c_{2}\left(E^{1 / 2}(t)+E^{2}(t)\right)\right] \frac{1}{k} E(t) \leq 0 .
$$

Thus, the exponential decay follows by choosing at the initial time:

$$
E^{1 / 2}(0)+E^{2}(0) \leq \frac{1}{2} \frac{c_{1}}{c_{2}}
$$

In fact, by continuity, for some time, $E(t) \leq E(0)$. But, this reinforces the previous condition so that the positive function in square brackets cannot decrease and is bounded from below by a positive constant for all times.

\section{Data Availability}

The data that support the findings of this study are available within the article.

\section{Conflicts of Interest}

The author declares that there are no conflicts of interest. 


\section{References}

[1] A. Giacobbe and G. Mulone, "Stability in the rotating Bénard problem and its optimal Lyapunov functions," Acta Applicandae Mathematicae, vol. 132, no. 1, 2014.

[2] A. Passerini, C. Ferrario, M. Růžička, and G. Thäter, "Theoretical results on steady convective flows between horizontal coaxial cylinders," SIAM Journal on Applied Mathematics, vol. 71, no. 2, pp. 465-486, 2011.

[3] A. Passerini and C. Ferrario, "A theoretical study of the first transition for the non-linear Stokes problem in a horizontal annulus," International Journal of Non-linear Mechanics, vol. 78, no. 1, pp. 1-8, 2015.

[4] A. De Martino and A. Passerini, "Existence and nonlinear stability of convective solutions for almost compressible fluids in Bénard problem," Journal of Mathematical Physics, vol. 60, no. 11, Article ID 113101, 2019.

[5] F. Crispo and P. Maremonti, "A high regularity result of solutions to modified p-Stokes equations," Nonlinear Analysis, vol. 118, pp. 97-129, 2015.

[6] H. Gouin and T. Ruggeri, "A consistent thermodynamical model of incompressible media as limit case of quasi-thermalincompressible materials," International Journal of NonLinear Mechanics, vol. 47, no. 6, pp. 688-693, 2012.

[7] A. Passerini and T. Ruggeri, "The Bénard problem for quasithermal-incompressible materials: a linear analysis," International Journal of Non-Linear Mechanics, vol. 67, no. 1, pp. 178-185, 2014.

[8] S. Gatti and E. Vuk, "Singular limit of equations for linear viscoelastic fluids with periodic boundary conditions," International Journal of Non-Linear Mechanics, vol. 41, no. 4, pp. 518-526, 2006.

[9] A. Corli and A. Passerini, "The Bénard problem for slightly compressible materials: existence and linear instability," Mediterranean Journal of Mathematics, vol. 16, no. 1, 2019.

[10] G. P. Galdi, "Non-linear stability of the magnetic Bénard problem via a generalized energy method," Archive for $\mathrm{Ra}$ tional Mechanics and Analysis, vol. 87, no. 2, pp. 167-186, 1985.

[11] G. P. Galdi and M. Padula, "A new approach to energy theory in the stability of fluid motion," Archive for Rational Mechanics and Analysis, vol. 110, no. 3, pp. 187-286, 1990.

[12] A. De Martino and A. Passerini, "A Lorenz model for almost compressible fluids," Mediterranean Journal of Mathematics, vol. 17, no. 1, p. 12, 2020.

[13] G. P. Galdi, An Introduction to the Mathematical Theory of the Navier-Stokes Equation, Springer, New York, NY, USA, 1998.

[14] O. Ladyzhenskaya, The Mathematical Theory of Viscous Incompressible Fluids, Gordon \& Breach, London, UK, 1963.

[15] G. Iooss, "Théorie non linéaire de la stabilité des écoulements laminaires dans le cas de "l'échange des stabilités," Archive for Rational Mechanics and Analysis, vol. 40, no. 3, pp. 166-208, 1971.

[16] M. Schechter, "Coerciveness in $L^{p}$," Transactions of the American Mathematical Society, vol. 107, no. 1, pp. 10-29, 1963. 